\title{
Viral Hepatitis and Agranulocytosis
}

\author{
Marigowda Nagaraju, MB, MRCP, Sigmund Weitzman, MD and Gregory \\ Baumann, MD
}

Transient hematopoietic aberrations are often found in association with viral hepatitis but are usually considered to be clinically insignificant. However, these changes are occasionally severe and sometimes fatal. Of the various hematologic manifestations which have been reported, agranulocytosis is very rare. Only two case reports are previously described in the literature $(1,2)$.

We have described here two cases of viral hepatitis with agranulocytosis. The bone marrow examination in both these patients showed hypercellularity.

\section{CASE REPORTS}

\section{Case One}

An 18-year-old white male (C.R., WCGH 472170) was admitted to Wayne County General Hospital on October 27,1971 , because of jaundice of 2 weeks' duration. He was well until 2 weeks before the onset of jaundice, when he experienced anorexia, nausea, and progressively increasing weakness. With the onset of jaundice he also noticed pruritus. Both gradually increased in severity. On the night before admission, fever, profuse perspiration, sore throat, and dry cough were noticed. He admitted to heavy wine drinking which he stopped after the onset of the present illness.

He denied oral or parenteral drug abuse, although he was closely associated with heroin addicts. Two of his friends were hospitalized because of hepatitis. He denied exposure to hepatotoxic drugs.

Trimeprazine, $2.5 \mathrm{mg}$ tablets, was prescribed by his family physician for nausea 4 weeks before hospitalization. $\mathrm{He}$ took only 2 or 3 tablets and discontinued the medication as he had no relief of symptoms.

Physical examination disclosed a well-developed white

From the Departments of Medicine, Wayne County General Hospital, Eloise, Michigan and University of Michigan Medical Center, Ann Arbor, Michigan.

Address for reprint requests: Dr. Marigowda Nagaraju, Department of Medicine, Wayne County General Hospital, Eloise, MI 48132. male with scleral icterus. Temperature was $102.8^{\circ} \mathrm{F}$. A few petechiae and excoriations were present on the extremities. The pharynx and tonsils were deeply injected, with small amounts of exudate behind the left tonsil. Lymph nodes in the anterior cervical regions were enlarged. The liver was palpable $3 \mathrm{~cm}$ below the right costal margin and was tender. The spleen was not palpable. Examination of other systems was unremarkable except for an ejection systolic murmur of 11/V11 intensity heard over the left sternal border.

Laboratory Data. Laboratory studies were as follows: hemoglobin, $15.5 \mathrm{~g} \%$; hematocrit, $46.6 \%$; WBC total count, $5200 / \mathrm{cu} \mathrm{mm}$, differential: nonsegmented polymorphs $2 \%$, lymphocytes $49 \%$, monocytes $48 \%$, eosinophils $1 \%$; platelets $210,000 / \mathrm{cu} \mathrm{mm}$; prothrombin time $82 \%$ of normal. Bilirubin was $13.7 \mathrm{mg} \%$; SGOT, $396 \mathrm{IU}$; alkaline phosphatase, $120 \mathrm{IU}$; total protein, $7.2 \mathrm{~g} \%$; albumin, 3.5 $\mathrm{g} \%$; globulin, $3.7 \mathrm{~g} \%, \alpha_{1} 6.4 \%, \alpha_{2} 10 \%, \beta 15.1, \gamma 20 \%$. Hepatitis-associated antigen was positive (counter electrophoresis method). Multiple blood cultures were negative. Heterophil antibody and antinuclear antibodies were also negative. Streptolysin $O$ titer was 166 Todd units. Urinalysis was normal. Throat culture grew normal bacterial flora of the mouth.

Bone marrow showed hypercellularity and maturation arrest of myeloid leukogenesis at the promyelocyte stage (Figure 1).

Liver biopsy demonstrated acute hepatitis with interportal bridging (Figure 2).

Hospital Course. Despite negative blood cultures, the patient was treated with parenteral antibiotics (cephalothin, $2 \mathrm{~g}$, at 6-hour intervals and gentamycin, $75 \mathrm{mg}$, at 8 hour intervals) because of fever and agranulocytosis. Fever subsided 3 days after treatment. Symptoms, liver function tests, and peripheral blood examination showed steady improvement. At the end of 2 weeks he was asymptomatic. Laboratory examination showed bilirubin, $2.1 \mathrm{mg} \%$; SGOT, $104 \mathrm{IU}$; WBC total count, 12,300/cu mm, metamyelocytes $1 \%$, nonsegmented neutrophils $30 \%$, segmented neutrophils $23 \%$, lymphocytes $39 \%$, monocytes $7 \%$. He was discharged from the hospital.

When he was seen in the outpatient department 4 weeks later, he was asymptomatic. Laboratory studies showed the 


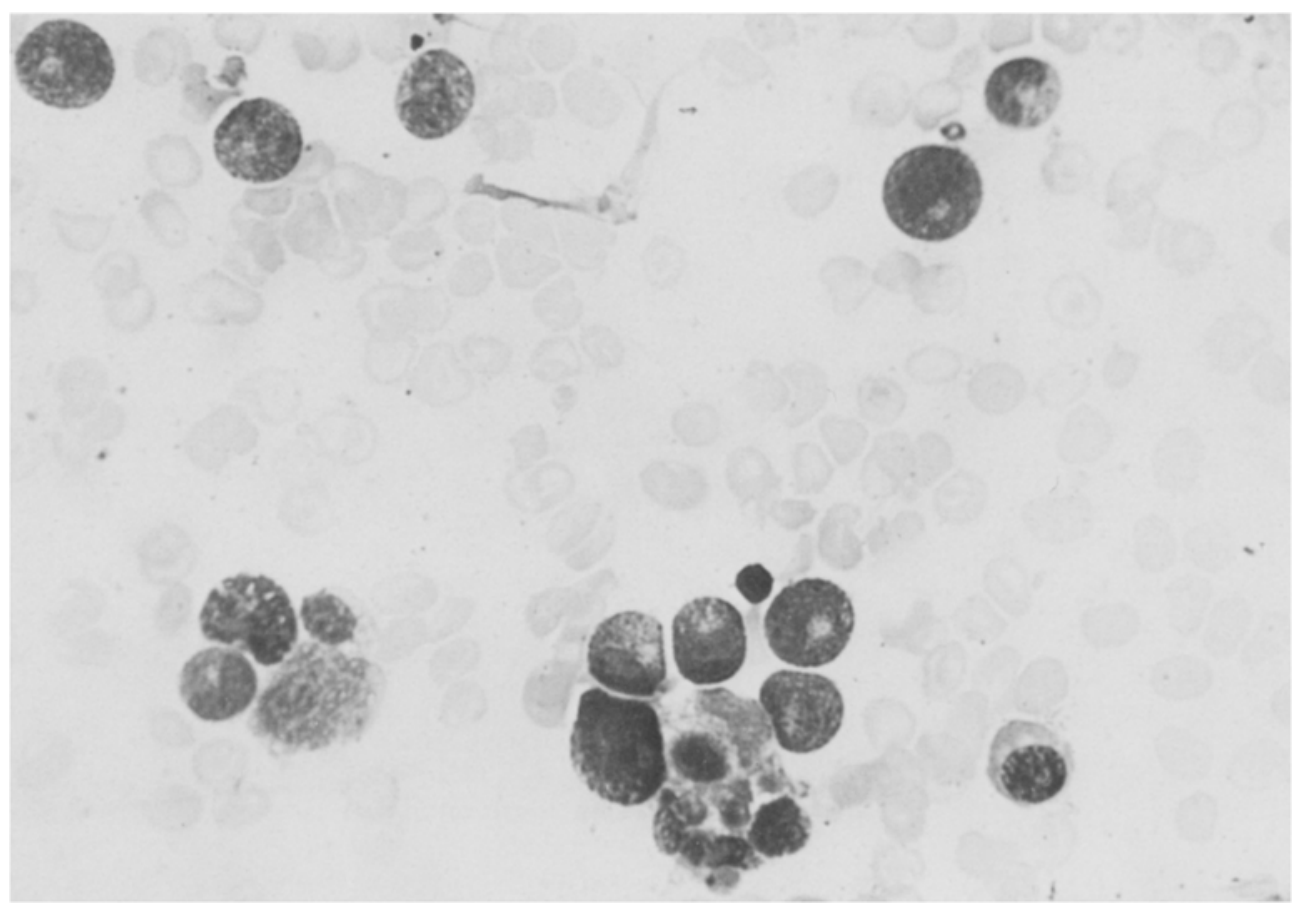

Fig 1. Myelogenesis arrested at promyelocyte stage. Note absence of polymorphonuclear leukocytes. $(H \& E, \times 320)$

following: bilirubin; $1.0 \mathrm{mg} \%$; SGOT; $38 \mathrm{IU}$, alkaline phosphatase, $70 \mathrm{IU}$; WBC total count, $7200 / \mathrm{cu} \mathrm{mm}$, nonsegmented neutrophils $17 \%$, segmented neutrophils $36 \%$, lymphocytes $33 \%$, monocytes $9 \%$, basophils $1 \%$, eosinophils $4 \%$.

\section{Case Two}

A 22-year-old white male (D.I., UMMC 012947) was admitted to University of Michigan Medical Center on October 20,1971, because of jaundice of 4 weeks' duration.

In 1961 splenectomy had been performed for idiopathic thrombocytopenia unresponsive to medical treatment. The patient had had many episodes of upper and lower respiratory tract infection. In 1962 a diagnosis of hypogammaglobulinemia was confirmed and the patient had been followed at our institution since that time until this current admission. One month prior to this admission he noticed nausea, malaise, arthralgia, jaundice, and abdominal pain with occasional vomiting. Laboratory studies performed by a local physician at that time revealed hematocrit, $42 \%$; WBC total count and differential count were normal. Bilirubin was $3.9 \mathrm{mg} \%$ and SGOT was $414 \mathrm{IU}$. Serum albumin was $3.4 \mathrm{~g} \%$ and globulin $2.9 \mathrm{~g} \%$.

Three weeks later the patient developed fever and his liver function tests showed deterioration. He was admitted to another hospital where he was given penicillin, 1 million units at 6-hour intervals IV, but his clinical condition continued to deteriorate. He was transferred to University Hospital. He denied use of heroin or other addictive drugs or contact with patients with hepatitis or exposure to hepatotoxic drugs. He admitted to drinking heavily before the onset of present illness.

Physical examination revealed a well-developed white male in moderate distress. He was deeply jaundiced, with a temperature of $101^{\circ} \mathrm{F}$, regular pulse 120 beats $/ \mathrm{min}$, and blood pressure of $140 / 90 \mathrm{mmHg}$. The liver was not palpable. Examination of other systems was unremarkable.

Laboratory Data. Laboratory studies were as follows: hemoglobin, $11.7 \mathrm{~g} \%$; hematocrit, 37.3\%; WBC total count, $7000 / \mathrm{cu} \mathrm{mm}$. The differential showed nonsegmented neutrophils $2 \%$, monocytes $51 \%$, lymphocytes $47 \%$, platelet count $402,000 / \mathrm{cu} \mathrm{mm}$. Bilirubin was $12.4 \mathrm{mg} \%$; SGOT, $510 \mathrm{IU}$; alkaline phosphatase, $124 \mathrm{IU}$; prothrombin time, 15.5 seconds with control of 10.5 seconds.

Hepatitis-associated antigen, antinuclear antibody, multiple blood cultures, heterophil antibody, and mumps antibody were all negative. Protein electrophoresis revealed total protein, $4.29 \mathrm{~g} \%$; albumin, $2.14 \mathrm{~g} \% ; \alpha_{1} 9.1 \%, \alpha_{2} 13.6 \%$, 
Fig 2. Hepatitis with interportal bridging. $(H \& E, \times 80)$

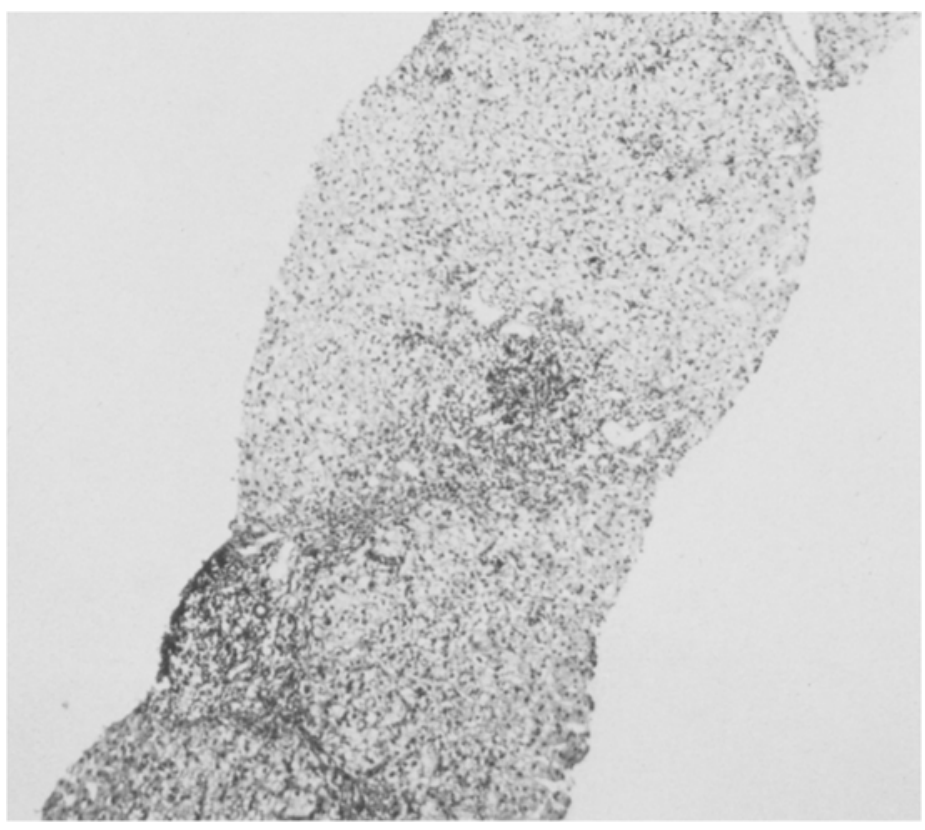

$315.2 \%, \gamma 12.1 \%$. Immunoelectrophoresis demonstrated total gammaglobulin $180 \mathrm{mg} \%, \operatorname{IgA}=0, \operatorname{IgG}=145 \mathrm{mg} \%$, $\operatorname{IgM}=35 \mathrm{mg} \%$.

Bone marrow showed hypercellularity and granulocytic hyperplasia with maturation arrest at the myelocyte stage (Figure 3).

Hospital Course. The patient was treated with antibiotics (cephalothin, $2 \mathrm{~g} \mathrm{IV}$, at 6-hour intervals and gentamycin, $75 \mathrm{mg}$, at 8 -hour intervais) and prednisone, $60 \mathrm{mg}$ daily, in addition to supportive therapy. There was some improvement in the clinical condition. Peripheral blood examination showed increasing granulocyte count.

At the end of 1 week hemoglobin was $10.6 \mathrm{~g}$; WBC $13,600 / \mathrm{cu} \mathrm{mm}$, nonsegmented neutrophils $5 \%$, segmented neutrophils $50 \%$, lymphocytes $34 \%$, monocytes $9 \%$, eosinophils $2 \%$, platelets $915,000 / \mathrm{cu} \mathrm{mm}$.

During the second week of hospitalization he developed recurrent episodes of gastrointestinal hemorrhage requiring multiple blood transfusions. Upper gastrointestinal tract $\mathrm{x}-$ rays showed thickening of the mucosal folds of small bowel compatible with submucosal edema. The patient developed oliguric renal failure and died on the 22 nd hospital day.

Two days before death, peripheral blood examination revealed WBC total count 14,800 , the differential was nonsegmented neutrophils $2 \%$, segmented neutrophils $64 \%$, monocytes $7 \%$, lymphocytes $27 \%$.

Autopsy examination revealed a small liver. Histologic examination of the liver showed submassive hepatic necrosis (Figure 4).

\section{DISCUSSION}

The clinical course, laboratory studies, and histologic features in these two patients are those of acute viral hepatitis. The first patient had positive hepatitis-associated antigen, indicating the possibility of parenteral transmission of virus. Despite the absence of hepatitisassociated antigen in the second patient, it is still possible that he had hepatitis $B$, especially in view of the multiple injections he had received during the course of the therapy for his hypogammaglobulinemia. The hypogammaglobulinemia is of interest in that Janeway and Gitlin (3) suggested that patients with deficient gammaglobulin may be more susceptible to the destructive effects of hepatitis virus. Similarly Good and Page (4) reported 2 patients with agammaglobulinemia who died with viral hepatitis.

Our second patient also had splenectomy 10 


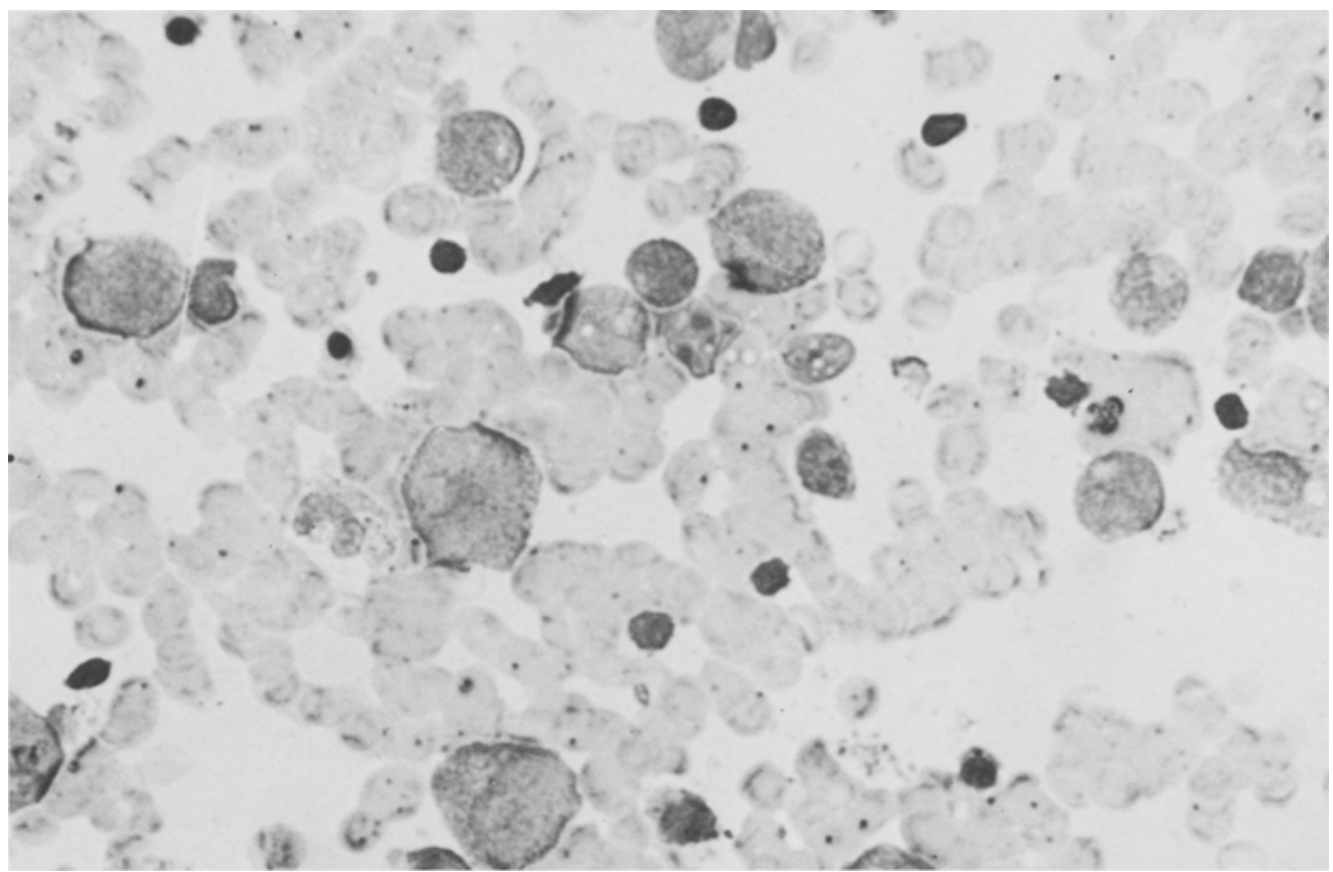

Fig 3. Maturation arrest at myelocytic stage. $(\mathrm{H} \& \mathrm{E}, \times 320)$

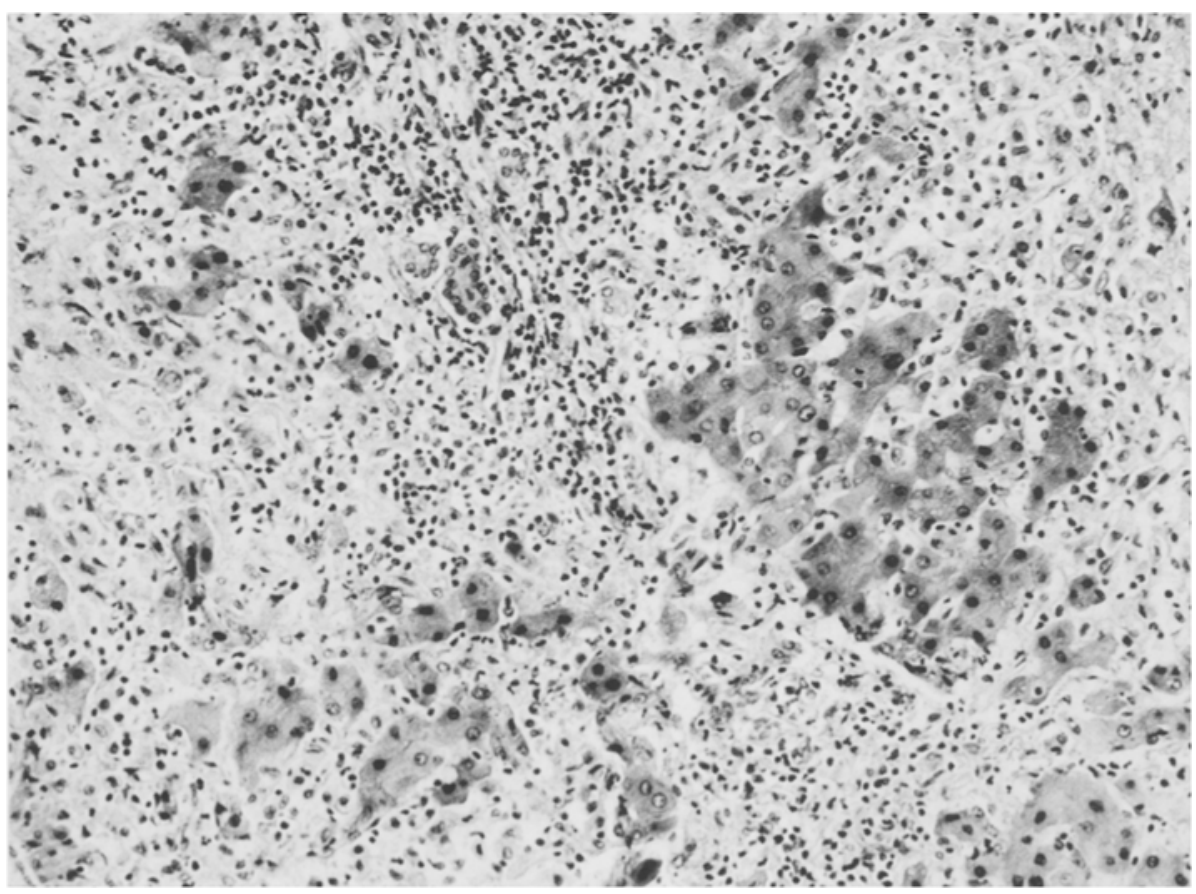

Fig 4. Fulminant hepatitis with distortion of lobular architecture and a few normal liver cells. $(H \& E, \times 160)$ 
years prior to the onset of hepatitis, but the role of splenectomy in the course of the disease is not clear. Stone et al (5) have noted $80 \%$ mortality in patients who developed hepatitis following splenectomy. The interval between splenectomy and onset of hepatitis was found to vary from 1 to 6 months. Horan and Colebatch (6) studied patients after splenectomy and found that $80 \%$ of infections occur within the first 2 years after splenectomy and that there was a tendency toward recurrent episodes of infection. It is possible in our patient that the additive effects of splenectomy and hypogammaglobulinemia might have caused the fatal outcome.

Phenothiazines are known to produce agranulocytosis. Pisciotta (7) pointed out that when blood dyscrasia was associated with exposure to chlorpromazine, the drug had usually been used for relatively long periods of time. Furthermore, there often was a latent period of time of 20 days or longer before the leukocyte count dropped. At the height of granulocytopenia the bone marrow was found to be markedly hypocellular. Such characteristics suggest a dose-related toxic action (8).

In support of the theory of dose-related toxic reaction Brachman et al (9) reported a case of agranulocytosis due to ingestion of $1030 \mathrm{mg}$ of trimeprazine. We do not believe that the doserelated toxic reaction was operating in our first case as he took only $7.5 \mathrm{mg}$ and his marrow was hypercellular at the height of agranulocytosis. We cannot exclude the possibility of an idiosyncratic reaction which was not dose-related.

Chronic alcoholics have been shown to have decreased marrow reserves of granulocytes, and septicemia may produce leukopenia with decrease in total cellularity of bone marrow and maturation arrest (10). This was only temporary; 48 hours later the bone marrow was shown to be full of granulocytes and peripheral blood rebounded with excessive leukocytes. This kind of response was not observed in our patients. Furthermore, the histology of liver tissue in the patients described did not show the changes of chronic alcoholism.
The hematopoietic changes seen in association with viral hepatitis are both quantitative and qualitative. The quantitative changes are common and include early leukopenia (12), thrombocytopenia, pancytopenia (13), and aplastic anemia (14). The qualitative changes are infrequent and include atypical lymphocytes, macrocytosis, decreased red cell survival (13), and hemolytic anemia. Erythroid aplasia has been noted after halothane hepatitis (15).

The pathogenesis of these hematopoietic changes remains largely unexplained. Autoimmune mechanism, failure of the liver to detoxify a myelosuppressive substance, and damage to the marrow by virus have all been postulated (16). Boxer et al have demonstrated the presence of antineutrophil antibody in apatient with neutropenia associated with chronic active hepatitis (17). Although this possibility cannot be ruled out in our patients as we did not test the serum of our patients, we feel that the pathogenesis of the disease process in active chronic hepatitis is different from that of viral hepatitis.

In experimental animals infected with MHV-3 virus, Piazza et al have shown hypoplasia of the erythroid series in the bone marrow and also marked delay in maturation of myelopoietic series and megakaryocytes, which may explain the anemia, granulopenia, and thrombocytopenia, respectively (18). The observations of Mella and Lang have been of greater interest. He has shown that serum of patients with infectious hepatitis, both in acute and convalescent phases, produces chromosomal abnormalities in the cultured leukocytes from normal persons (19). This lends credence to the hypothesis that aplastic anemia may be due to chromosomal damage. They have also shown that this serum inhibits mitosis of leukocytes from normal subjects. Although the nature of the substance that causes inhibition of maturation of leukocytes is not known, it is likely that it is present in the serum of hepatitis patients. This substance may be the virus or vi- 
ral particle or an unidentified serum factor. This is the plausible explanation that we could offer for the maturation arrest. It should be noted that these changes are only temporary. In the first patient the improvement was rapid, and his peripheral blood was normal before the signs of hepatitis cleared. The second patient with hypogammaglobulinemia succumbed to the complications of hepatitis. The death of this patient may offer some support to the view of Janeway and Gitlin that the patients with deficient gammaglobulins are more susceptible to the effects of hepatitis virus infection.

\section{ACKNOWLEDGMENTS}

The authors wish to thank Dr. B. Bercu, Director of Medicine and Dr. R.R. Joseph, Director of Gastroenterology, Wayne County General Hospital for their encouragement and Dr. N.A. Nelson, hematologist, Wayne County General Hospital, for the bone marrow report and criticism.

\section{REFERENCES}

1. Sabrazes J, Saric R: Angines Lymphomonocytaires, Agranulocytoses. Leucemies Leucopeniques, Paris, 1955, p 251

2. Dische FE, Colding JR: Agranulocytosis associated with infective hepatitis. Br Med J 2:738740, 1957

3. Janeway CA, Gitlin D: The gamma globulins, Advances in Pediatrics. Edited by SZ Levine. Chicago, Yearbook Publishers Inc, 1957, Vol 9, p 65-136

4. Good RA, Page AR: Fatal complications of virus hepatitis in two patients with agammaglobulinemia. Am J Med 29:804-810, 1960

5. Stone HH, Stanley DG, DeJarnette: Postsplenectomy viral hepatitis. JAMA 199:187189,1967
6. Horan M, Colebatch JH: Relation between splenectomy and subsequent infection: a clinical study. Arch Dis Child 37:398-414, 1962

7. Pisciotta AV: Agranulocytosis induced by certain phenothiazine derivatives. JAMA 208:1862-1868, 1969

8. Wintrobe $\mathrm{MH}$ : Agranulocytosis and infectious mononucleosis, Clinical Hematology. Philadelphia, Lea \& Febiger, 1967, p 1220

9. Brachman PS, McCreary TW, Florence R: Agranulocytosis induced by trimeprazine. N Engl J Med 260:378-380, 1959

10. Schick G, Virks J: Agranulocytosis associated with chlorpromazine therapy: a report of a case and review of literature. $\mathrm{N}$ Engl J Med 255:798-802, 1956

11. McFarland W, Libre EP: Abnormal leukocyte response in alcoholism. Ann Intern Med 59:865-877, 1963

12. Havens WP Jr, Marck RE: The leukocyte response of patients with experimentally induced infectious hepatitis. Am J Med Sci 212:129 138,1946

13. Kivel RM: Hematologic aspects of acute viral hepatitis. Am J Dig Dis 6:1017-1031, 1961

14. Rubin E, Gottlieb C, Vogel P: Syndrome of hepatitis and aplastic anemia. Am J Med 45:88-97, 1968

15. Jurgensen JC, Abraham JP, Hardy WW: Erythroid aplasia after halothane hepatitis: report of a case. Am J Dig Dis 15:577-581, 1970

16. Editorial: Infectious hepatitis and aplastic anemia. Lancet 1:844-845, 1971

17. Boxer LA, Yokoyama M, Wieber RA: Autoimmune neutropenia associated with chronic active hepatitis. Am J Med 52:279-282, 1972

18. Piazza M, Piccinino F, Matano F: Hematological changes in viral (MHV-3) murine hepatitis. Nature 205:1034-1035, 1965

19. Mella B, Lang DJ: Leukocyte mitosis: suppression in vitro associated with acute infectious hepatitis. Science 155:80-81, 1967 